EPJ Web of Conferences 66, 02034 (2014)

DOI: $10.1051 /$ epjconf/ 20146602034

(C) Owned by the authors, published by EDP Sciences, 2014

\title{
A new spin-oriented nuclei facility: POLAREX
}

A. Etilé ${ }^{1, a}$, A. Astier ${ }^{1}$, G. Audi ${ }^{1}$, S. Cabaret ${ }^{1}$, C. Gaulard ${ }^{1}$, G. Georgiev ${ }^{1}$, F. Ibrahim ${ }^{2}$, J. Nikolov $^{3}$, L. Risegari ${ }^{4}$, S. Roccia ${ }^{1}$, G. Simpson ${ }^{5}$, J.R. Stone ${ }^{6,7}$, N.J. Stone ${ }^{6,7}$, D. Verney ${ }^{2}$, and M. Veskovic ${ }^{3}$

${ }^{1}$ CSNSM, Université Paris-Sud/CNRS-IN2P3, Bât. 104 et 108, 91405 Orsay Campus, France

${ }^{2}$ IPNO, Université Paris-Sud/CNRS-IN2P3, 91406 Orsay Campus, France

${ }^{3}$ University of Novi Sad, Faculty of Sciences, Dep. of Physics, Trg Dositeja Obradovica 3, Novi Sad, Serbia

${ }^{4}$ Laboratoire Commun de Métrologie LNE-CNAM, 61, rue du Landy, 93210 La Plaine Saint-Denis, France

${ }^{5}$ LPSC, Université Joseph Fourier/CNRS-IN2P3, Grenoble INP, 38026 Grenoble Cedex, France

${ }^{6}$ Department of Physics and Astronomy, University of Tennessee, Knoxville, Tennessee 37996, USA

${ }^{7}$ Department of Physics, Oxford University, Parks Road, Oxford OX1 3PU, Oxford, UK

\begin{abstract}
.
Using the On-Line Nuclear Orientation method, POLAREX (POLARization of EXotic nuclei) is a new facility allowing to study the anisotropic decay of spin-oriented nuclei. Based on the combination of on-line implantation of radioactive nuclei with Low Temperature Nuclear Orientation technique and Nuclear Magnetic Resonance, POLAREX allows to measure nuclear electromagnetic moments and ground-state spins, in the aim to get information about the wave function composition of the nuclear state. Polarized nuclei can also be used to study fundamental interactions involving nuclear $\beta$-decay asymmetries. The POLAREX infrastructure will be installed at Accélérateur Linéaire auprés du Tandem d'Orsay in order to study neutron-rich nuclei, some of which have not been studied yet. Will be presented here, all the possibilities of this new facility and a non exhaustive scientific program.
\end{abstract}

\section{Introduction}

Even though nuclear physics is now a centenary science, we still don't have a fundamental description of the nuclear forces and no comprehension of their nature. Indeed, the nucleus is a complex quantum bound system ; despite the repulsive Coulomb force, the nucleons moving and interacting with each others, are kept bound together by the short-range strong nuclear force. In order to test, develop and improve our theoretical models we have to measure fundamental nuclear properties.

Decades ago, different nuclear models were built, essentially based on study of nuclei close to the valley of stability. However, their predictive power is inadequate when applied to nuclei further from stability. That's why one of the keys to understand nuclear interaction is to study exotic nuclei. While accelerator facilities are able to produce more and more exotic nuclei, the POLAREX (POLARization of EXotic nuclei) [1] project has been developped in the willingness to participate to the study of nuclei under extreme conditions (very low temperature, very high magnetic field).

\footnotetext{
ae-mail: etile@csnsm.in2p3.fr
}

This is an Open Access article distributed under the terms of the Creative Commons Attribution License 2.0, which permits unrestricted use, distribution, and reproduction in any medium, provided the original work is properly cited. 
The low temperature nuclear orientation (LTNO) [2] is obtained by having an ensemble of nuclei cooled down to $\sim 10 \mathrm{mK}$ with a ${ }^{3} \mathrm{He}-{ }^{4} \mathrm{He}$ dilution refrigerator under an external magnetic field $\left(\boldsymbol{B}_{\text {app }}\right)$. The nuclei are in addition implanted into a ferromagnetic foil attached to the cold finger in the cryostat, and oriented through the then created internal hyperfine field $\left(\mathbf{B}_{\boldsymbol{h f}}\right)$.

The measurements can be made according to two modes: off-line and on-line modes. An online measurement means that the nuclei to be oriented are produced by an accelerator and directly implanted inside the dilution refrigerator.

Taking advantage of the possibility to be installed at ALTO (Accélérateur Linéaire auprés du Tandem d'Orsay) [3], POLAREX will allow to measure basic and essential characteristics of neutronrich nuclei provided by the accelerator (for instance spins), but also finer ones such as electromagnetic moments. Studying the angular distribution of the radiations emitted by an ensemble of oriented nuclei, the LTNO method allows much more than nuclear properties measurements, by being at the intersection of two major fields in physics : nuclear physics ans solid states physics.

\section{Principle of operation}

In order to get an ensemble of spin-oriented nuclei, two conditions are necessary : low temperature and a high magnetic field or alternatively an electric field gradient (EFG). Indeed, because of the interaction between its nuclear magnetic moment $\boldsymbol{\mu}$ and an external field, a non-zero nuclear spin $(I)$ nucleus experiences the Zeeman splitting. In the same way nuclei with spins $>\frac{1}{2}$ have their electric quadrupole moments interacting with the EFG of the nuclear surroundings. The $2 \mathrm{I}+1$ hyperfine nuclear substates are then populated according to Boltzmann distribution :

$$
p(m)=\frac{e^{-\frac{m \Delta E}{k T}}}{\sum_{m} e^{-\frac{m \Delta E}{k T}}},
$$

with $p(m)$ the population of the $m$ (projection of the angular momentum) Zeeman sublevel, $\Delta E=\mu B / I$ the energy difference between two substates with $\mathbf{B}=\boldsymbol{B}_{\text {app }}+\boldsymbol{B}_{\boldsymbol{h f}}$ the total magnetic field, $k$ the Bolztmann constant and $T$ the absolute temperature. When the temperature $T$ is sufficiently low i.e. when the thermal energy is of the same order as the Zeeman splitting energy $\Delta E$, a spontaneous polarization can be observed.

With Polarex, the radioactive nuclei implanted into a ferromagnetic host foil held at $\sim 10 \mathrm{mK}$, experience a high degree of polarization due to the hyperfine interaction. Under an external magnetic field $\left(\boldsymbol{B}_{\boldsymbol{a p p}} \sim 0.5 T\right)$ nuclei are oriented through the internal hyperfine field $\left(\boldsymbol{B}_{\boldsymbol{h f}} \sim 100 T\right)$.

While at room temperature, the decay products emission is isotropic, spins of oriented nuclei present a preferential direction with respect to $\boldsymbol{B}_{\text {app }}$ (or the EFG) : emission becomes anisotropic at low temperature (see figure 1). The anisotropic shape $W(\theta)=\frac{N_{\text {cold }}(\theta)}{N_{\text {warm }}(\theta)}$ of the decay products is defined as the ratio of the count rate $N$ at a given angle $\theta$ measured at two different temperatures : cold $(\sim 10 \mathrm{mK})$ and warm $(\sim 1 \mathrm{~K})$. It is related to important characteristics of the studied nucleus : spins of the involved states, transition multipolarity $(\lambda)$, parity admixture and depends on the total magnetic field $(\mathbf{B})$ and the temperature $(T)$. Those parameters can be extracted from the following relation :

$$
W(\theta)=1+f \sum_{\lambda} A_{\lambda} B_{\lambda} U_{\lambda} Q_{\lambda} P_{\lambda}(\cos \theta)
$$

where $f$ represents the fraction of nuclei experiencing the hyperfine field, $A_{\lambda}$ are the angular distribution factors, $B_{\lambda}$ the orientation parameters, $U_{\lambda}$ the deorientation coefficients, $Q_{\lambda}$ geometrical parameters and $P_{\lambda}(\cos \theta)$ stand for order $\lambda$ Legendre polynomial. 


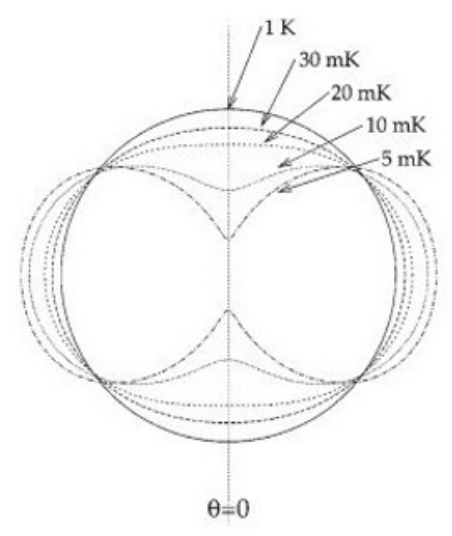

Figure 1. Example of shape of the anisotropy of the emitted gammas as a function of the temperature (from [4])

The main constraint in this method is due to the spin lattice relaxation time of the implanted nuclei in the host. It typically limits our choice to nuclei with lifetime $>1 s$. The sample temperature is monitored with a needle of ${ }^{60} \mathrm{CoCo}(\mathrm{hcp})$ single crystal used as a NO thermometer [? ]. Just like our sample it is stuck on the cold finger with a good thermal contact.

Coupled to NMR (Nuclear Magnetic Resonance), the OLNO (On-Line Nuclear Orientation) method allows to measure nuclear magnetic moments. A modulated RF (Radio Frequency) field is produced by a resonance coil placed inside the cryostat, with axis normal to the applied field $\left(\boldsymbol{B}_{\text {app }}\right)$. When the frequency matches the hyperfine splitting energy, resulting from the resonant absorption one can observe a reduction of the anisotropy and get a precise measurement of the $g$-factor $\left(\mu=g \mu_{N} I\right.$, with $\mu_{N}$ the nuclear magneton). The nuclear magnetic moment of the oriented state is determined when the resonant frequency $v_{0}$ is matched :

$$
v_{0}=\frac{|\mu|}{I h}\left(B_{h f}+B_{a p p}(1+K)\right)
$$

with $K$ the Knight shift.

\section{Scientific program and outlooks}

\subsection{Nuclear structure...}

The OLNO method and equation (2) are valid for different kinds of radiations : gamma rays, beta and alpha decays. The POLAREX facility is mainly dedicated to nuclear magnetic moment measurements, a fine characteristic of the nucleus that can be directly compared with predictions. Nuclear moments provide important information on the nuclear state wave function : magnetic dipole moment being sensitive to the single particle aspect, while electric quadrupole moment is related to the collective behaviour of nuclei. In addition to their role as input parameters in theoretical models, they allow to confirm hypotheses based on indirect experimental measurements.

The originality of POLAREX comes from the beam line accessing by the bottom, allowing to fit more detectors, thus providing higher precision measurements and a better accuracy. It also opens the way to electric quadrupole moment measurement which is a more difficult and challenging one. 
Furthermore, basic parameters such as level spins in the daughter nucleus or finer characteristics like parity admixture can be accessed, also important aspects of nuclear structure can be learnt from gamma-multipolarity or decay parameters.

Complementary to the only other On-Line Nuclear Orientation device NICOLE@ISOLDE [5], POLAREX@ALTO will be a unique worldwide facility to study neutron-rich nuclei. Indeed, among the ALTO production there are about 300 candidates that fulfill the conditions for an OLNO experiment, amongst which 200 have unmeasured nuclear magnetic moment. The first off-line studies will concentrate on light lanthanides a still not-well known region where hyperfine interactions are strong. Then, nuclear structure studies around the doubly magic nuclei ${ }^{78} \mathrm{Ni}$ and ${ }^{132} \mathrm{Sn}$ will initiate the on-line program.

\section{2 ....and beyond}

Since 1956, when Lee and Yang first observed the parity violation in an electroweak decay [6], a fundamental question remained unanswered : "why the total or partial parity violation is still not theoretically predicted?" In this context, LTNO is a great tool to probe symmetries in the nuclear interaction. The measured anisotropy shape of the emitted beta particles allows weak interaction tests such as CPT violation test, and an accurate determination of the $\beta$-asymmetry parameter [7].

As mentionned before, LTNO being at the overlapping between nuclear physics and solid state physics, important information in solid state physics can also be determined. A major one is the hyperfine field, one can go further determining spin lattice relaxation times, the hyperfine anomaly, or exploring magnetic structures. For instance, studies on lanthanides will provide the important solid state physics result of their hyperfine field which are still unknown.

Nuclear orientation being the most accurate way to determine very low temperature, this facility can also be used as a high precision tool for thermometry calibration, and studies on thermometers in high magnetic field, or under RF radiations.

\section{Conclusion}

A new LTNO facility, POLAREX, which already received several letters of intention will be installed at ALTO. Taking advantages of its particular geometry and the neutron-rich nuclides production of ALTO, it will be a unique facility for new experiments based on LTNO with a wide range of applications. The first experiment will be the nuclear magnetic moment measurement of ${ }^{125} \mathrm{Sb}$ as final commissioning.

\section{References}

[1] www.csnsm.in2p3.fr/polarex/

[2] N.J. Stone and H. Postma, Low Temperature Nuclear Orientation, North-Holland Publ. Comp., (1986)

[3] ipnweb.in2p3.fr/tandem-alto/

[4] W. D. Hutchison, Applications of low temperature nuclear orientation, Hyperfine Int., (2013).

[5] R. Eder et al., NICOLE : New On-Line Orientation Facility at ISOLDE/CERN, Hyperfine Interactions 60, (1990).

[6] T.D. Lee and N. Yang, Question of parity conservation in weak interactions, Phys. Rev. 104, (1956).

[7] F. Wauters et al., Precision measurements of the ${ }^{60}$ Co $\beta$-asymmetry parameter in search for tensor currents in weak interactions, Phys. Rev. C 82, (2010). 